\title{
Utilidad de la automonitorización en el tratamiento de la terapia con warfarina
}

\section{Usefulness of self-monitoring in warfarin therapy}

\author{
Guillermo Mora-Pabón ${ }^{\mathrm{a}, \mathrm{b}, \mathrm{c}, \mathrm{d}}$ \\ a Departamento de Medicina Interna, Universidad Nacional de Colombia, Bogotá, Colombia \\ b Fundación Santa Fe de Bogotá, Bogotá, Colombia \\ c Hospital Universitario Nacional, Bogotá, Colombia \\ 'Clínica San Rafael, Bogotá, Colombia
}

Recibido el 3 de octubre de 2016; aceptado el 6 de octubre de 2016

Disponible en Internet el 4 de noviembre de 2016

\section{Introducción}

Aunque la llegada de los anticoagulantes orales directos (DOAC, su sigla en inglés) es una realidad y ha cambiado el enfoque inicial del tratamiento y las complicaciones hemorrágicas en pacientes con fibrilación auricular, aún existen grupos de pacientes en quienes la warfarina sigue siendo la terapia de elección. Se incluyen aquellos con fibrilación auricular valvular (asociada a la estenosis mitral, prótesis valvulares mecánicas), insuficiencia renal con menos de $15 \mathrm{ml} / \mathrm{kg}$ de depuración de creatinina, o previamente bien controlados con warfarina. Las guías de manejo de fibrilación auricular aún recomiendan como una buena opción el inicio de warfarina (clase ı, nivel de evidencia A) en el paciente con alto riesgo embólico ${ }^{1}$.

Uno de los mayores inconvenientes del uso de warfarina es su interacción con los alimentos, otras medicaciones o estado intercurrente de otras enfermedades. Bien se sabe que muchas drogas pueden aumentar su efecto como antibióticos, diltiazem y antiarrítmicos comúnmente usados en fibrilación auricular como amiodarona o propafenona ${ }^{2}$. Por otra parte, otras drogas como rifampicina, barbitúricos, fenitoína, etc. pueden disminuir su efecto. Los cambios en el consumo de comidas con altas concentraciones de

Correo electrónico: gmorap@unal.edu.co vitamina $\mathrm{K}$ (vegetales verdes) pueden revertir los efectos anticoagulantes ${ }^{3}$. Condiciones médicas como: diarrea, falla cardiaca, fiebre, hipertiroidismo y hepatopatías pueden potenciar el efecto de la warfarina ${ }^{2}$. La eficacia de este medicamento y el riesgo de complicaciones hemorrágicas se relacionan con mantener un INR adecuado, el cual tiene un estrecho rango terapéutico ${ }^{4}$. Estos factores dificultan la selección de la dosis ideal de inicio, estabilización y mantenimiento de un efecto estable a largo término. Adicionalmente, está claro que el mantenimiento en el rango terapéutico del INR se traduce en mejores resultados tromboembólicos y hemorrágicos ${ }^{5}$.

Los sistemas de monitorización y el automanejo basado en estos resultados a cargo del propio paciente, pueden ofrecer ventajas sobre otras aproximaciones; en el primer caso el paciente determina su propio INR e informa al médico para definir cambios en la dosis. En el segundo, el paciente no solo determina su INR sino que hace el ajuste de la dosis de warfarina a partir de unos límites dados con antelación ${ }^{6}$. Esta forma de manejo ha surgido de los resultados de automonitorización de la glicemia y del cambio en las dosis de insulina, que han ido avanzando con gran éxito.

El uso de estos sistemas puede facilitar el suministro de warfarina en pacientes con dificultades para asistir a una clínica de anticoagulación e igualmente puede incrementar el número de veces que se determina el INR. Se ha encontrado que la frecuencia de la realización del INR es un factor 
predictivo positivo para mantenerlo en rango terapéutico ${ }^{5}$. Incluso, hay evidencia de que puede relacionarse con menores complicaciones.

La mayoría de pacientes anticoagulados con warfarina son tratados por sus propios médicos; no hay programas organizados de educación o seguimiento y hay pocos estudios que evalúen los resultados clínicos de estas poblaciones. Una guía que resumió cuatro series, encontró en 1.772 pacientes (2.293 paciente/año) un 7-8\% pacientes/año con complicaciones hemorrágicas mayores, de las cuales 0,9 eran fatales y $8,1 \%$ pacientes/año con eventos tromboembólicos y $15 \%$ pacientes/año con algún evento serio ${ }^{7}$.

La aparición de las clínicas de anticoagulación mejoró los resultados clínicos al mantener un mayor tiempo en rango terapéutico (TRT), al disminuir fenómenos trombóticos y hemorrágicos, y llevar así a una mejor relación costo-efectividad del tratamiento. Los estudios observacionales que se resumen en esta guía, muestran 13.475 casos (18.960 pacientes/año de seguimiento), 2,8\% pacientes/año de eventos hemorrágicos mayores ( 0,39 fatales) y $2,6 \%$ pacientes/año de eventos trombóticos ${ }^{7}$. Cuando se evalúan estudios que comparan el cuidado usual con el de las clínicas de anticoagulación, se evidencia con claridad la superioridad de estas últimas, llegando en la mayoría de casos a 0 fatalidades, entre 0 y 3,9\% eventos hemorrágicos pacientes/año y entre 0 y 3,5\% de eventos trombóticos ${ }^{8-11}$.

Los dispositivos para determinar el INR en forma ambulatoria se basan en la detección del coágulo usando tromboplastina como iniciador del mismo. Éstos han sido probados en diferentes escenarios y han demostrado exactitud y precisión ${ }^{12}$.

Los estudios que comparan la automonitorización con el cuidado usual, muestran una mejoría marcada en términos de TRT o eventos adversos. Los valores de TRT con la automonitorización oscilan entre un 57 y un $92 \%{ }^{13,14}$, en tanto que los resultados del cuidado usual son cercanos al 34 y $62,5 \%$. Los estudios que comparan la automonitorización y la autoterapia (modificación de la dosis de warfarina por el paciente de acuerdo con el resultado del INR), indican que el TRT oscila entre 56 y $93 \%{ }^{15,16}$, mientras que el cuidado usual está alrededor del 33\%. De igual forma, las complicaciones hemorrágicas fueron más comunes en el cuidado usual (2,2-12\% vs. $0-6,3 \%)$, así como lo fueron también los eventos trombóticos (3,6-13\% vs. 0,9-9\%) ${ }^{7}$. Recientemente, se publicó el resultado de una cohorte suiza de 1.140 pacientes (5.819 pacientes/año) en automonitorización seguidos en promedio por 4,3 años. La frecuencia de eventos trombóticos fue de $0,4(95 \%$ IC $0,2-0,6)$ y la de eventos hemorrágicos $1,1(95 \% I C \quad 0,9-1,5)$ por 100 pacientes/año ${ }^{17}$. En conclusión, la automonitorización se asocia con mejores resultados clínicos que el cuidado usual.

Cuando se compara la automonitorización con el tratamiento de pacientes en clínicas de anticoagulación, los resultados muestran que son tan buenos o en ocasiones mejores con la primera. Así mismo, señalan que el TRT en clínicas de anticoagulación oscila entre 65 y $80 \%{ }^{18,19}$, mientras que con el autocuidado están entre 55 y $93 \%$. Las complicaciones hemorrágicas estuvieron entre $0,4 \%$ para el autocuidado y 0 para las clínicas de anticoagulación. Por otra parte, los eventos embólicos rondaron el 0 y el $16 \%$ (pacientes/año) en las clínicas de anticoagulación y el $0 \%$ en los pacientes de automonitorización ${ }^{7}$. Aunque algunos de estos estudios fueron aleatorios, ninguno se designó claramente para definir cuál era la mejor estrategia de manejo ni controlaron correctamente variables de confusión como frecuencia de monitorización, cumplimiento, tipo de dispositivo usado o grado de educación del paciente.

Recientemente, un estudio japonés evaluó la automonitorización en pacientes que asistían a clínicas de anticoagulación y el TRT 12 meses antes y después del inicio de esta. En 148 pacientes se observó mayor TRT con la introducción de la automonitorización (51,9 $\pm 33 \%$ vs. $69,3 \pm$ $26,3 \% \mathrm{p}<0,001)$. El efecto fue mayor en aquellos que tenían TRT $<70 \%$ antes de la intervención. Los cambios fueron más notorios en los valores por debajo del INR terapéutico $(44,4$ $\pm 34,4 \%$ vs. $27,4 \pm 27,6 \% p<0,0001)$ que en aquellos por encima de este $(3,7 \pm 10,6 \% \text { vs. } 3,3 \pm 6,3 \% p=0,73)^{20}$. Sin embargo, la automonitorización no ha demostrado ser útil cuando se compara con clínicas de anticoagulación de alta calidad. Un estudio aleatorizado cotejó la automonitorización en 2.922 pacientes una vez a la semana o la monitorización una vez al mes en clínica de anticoagulación. Se siguieron entre 2 y 4,75 años (8.730 pacientes/año). Aunque hubo mayor TRT en la auto-monitorización no se registraron diferencias en el tiempo del primer evento trombótico, sangrado mayor o muerte ${ }^{21}$. Finalmente, una revisión sistemática encontró que la automonitorización y el automanejo de acuerdo con la automonitorización, pueden mejorar la calidad de la anticoagulación, con reducción en el RR 0,5 (IC 95\% 0,36-0,6) para eventos embólicos y de 0,64 (IC 95\% 0,46-0,89) para todas las causas de mortalidad. No hubo aumento en los eventos adversos, pero solo la mitad de los pacientes acepta este manejo ya sea por rechazo, exclusión por su médico de cabecera o inhabilidad para completar el entrenamiento ${ }^{22}$.

Así mismo, es importante conocer la exactitud de estas pruebas. En un estudio de 1.009 pacientes, el coeficiente de correlación global fue de 0,95 para CoaguCheck XS plus y de 0,88 para INRatio PT (los dos sistemas existentes en el país). Para INR $<2$ el coeficiente fue 0,92 y 0,81 respectivamente. Finalmente, con rangos de INR > 3, la correlación fue de 0,8 para CoaguCheck xs plus y de 0,54 para INRatio PT monitor ${ }^{23}$. Otro estudio con 4.326 pacientes no encontró diferencias entre CoaguCheck xs o INRatio $2^{24}$.

Como hallazgo importante, los pacientes que están con automonitorización tienen mejoría en su calidad de vida. Un estudio encontró que los pacientes preferían esta opción por menor tiempo de espera, menos dolor y necesidad de sangre, así como por resultados más rápidos ${ }^{25}$.

Al analizar la costo-efectividad de esta intervención existe información importante. Lo primero que se debe tener en mente es el costo de una anticoagulación subóptima. En un estudio económico sobre 2,3 millones de estadounidenses con fibrilación auricular, se determinó que si el 50\% de quienes reciben warfarina estuvieran bien tratados, se prevendrían 9.852 eventos embólicos y se ahorrarían 13.000 millones de dólares ${ }^{26}$. Sin embargo, los diferentes sistemas de salud no cubren los coagulómetros debido al costo de los aparatos y las tiras, al igual que del personal que entrena los pacientes. En un subestudio del GELIA ${ }^{27}$ se encontró reducción de los costos asociados a un $30 \%$ de minimización de complicaciones graves y a un $20 \%$ de 
eventos hemorrágicos intracraneanos letales observados con la automonitorización. El costo fue de 52.000 euros por año de vida ganado. Un estudio en el Reino Unido encontró que sobre 10 años de automonitorización se ahorrarían 1.187 libras esterlinas por persona tratada; en sus cálculos, si el sistema de salud comprara el coagulómetro, el costo del mismo se salvaría al final del segundo año de tratamiento, considerándose costo-efectivo. Recientemente se publicó una revisión sistemática de la automonitorización con evaluación económica, en la que se identificaron 26 estudios aleatorizados con 8.763 pacientes, la mayoría con CoaguCheck XS y S, y se determinó que la intervención es costo-efectiva si la automonitorización disminuye eventos trombóticos ${ }^{28}$.

Aunque la mayoría de estudios han comparado automonitorización con tratamiento estándar o en clínicas de anticoagulación con warfarina, un estudio hizo una comparación indirecta con dabigatrán y encontró resultados similares entre los dos grupos en cuanto a trombosis, sangrado y muerte ${ }^{29}$. Pese a ello, conviene tener en cuenta las limitaciones de este tipo de estudios.

No existe consenso sobre cuáles son los pacientes que pueden ser seleccionados para automonitorización/autotratamiento. Puede considerarse, no obstante, el éxito del programa de entrenamiento del estudio SMART ${ }^{30}$. Los pacientes podían ingresar al programa solo después de al menos doce meses de anticoagulación, tener el deseo (o de su cuidador) de participar en el programa de entrenamiento y aprobarlo. Más o menos un $75 \%$ de las personas aprobó esta fase; se resalta que en otro estudio solo lo hizo un $50 \%{ }^{31}$.

El programa del entrenamiento incluía aspectos teóricos de la anticoagulación, como monitorizar, frecuencia de la monitorización, problemas con esta monitorización, interacción de anticoagulantes con otras medicaciones, influencia de la nutrición, el alcohol y enfermedades intercurrentes en la anticoagulación, reconocimiento de complicaciones y concepto de INR objetivo e importancia de mantenerlo dentro del 0,5 del objetivo. Las sesiones prácticas incluían manipulación del coagulómetro, práctica de un test de coagulación, realizar un procedimiento de punción del dedo e identificar posibles fuentes de error y riesgo de resultados. Recientemente, un pequeño estudio de educación para automonitorización demostró la factibilidad del entrenamiento y el alto nivel de comodidad de los pacientes ${ }^{32}$.

Precisamente, los profesionales de la salud que entrenan a los pacientes para automonitorización, deben ser entrenados en estos temas. En los programas actuales este entrenamiento dura un día e incluye aspectos teóricos y farmacéuticos de los anticoagulantes, uso del equipo y una sesión práctica ${ }^{33}$. Los centros de entrenamiento deben estar disponibles para consultas del paciente y monitorizar uno a tres resultados del INR al mes. Aunque no hay claridad en el número de pacientes que se entrenan por sesión, la experiencia alemana sugiere un número reducido (3-5 pacientes $)^{7}$. Por otra parte, para proveer garantías metodológicas y de funcionamiento, los coagulómetros se evalúan al menos una vez al año.

Para finalizar, es importante resaltar que la automonitorización está claramente indicada en diferentes guías internacionales de manejo ambulatorio del paciente anticoagulado $2,7,34$.

\section{Recomendaciones}

\section{Clase I}

- La automonitorización está indicada en pacientes (o su cuidador) altamente motivados que han aprobado el entrenamiento y que no pueden asistir a clínicas de anticoagulación (nivel de evidencia $B$ ).

\section{Clase II A}

La automonitorización puede recomendarse en pacientes (o su cuidador) altamente motivados que han aprobado el entrenamiento como alternativa a una clínica de anticoagulación (nivel de evidencia A).

\section{Clase III}

- La automonitorización no está indicada en pacientes (o cuidadores) que por problemas visuales, motrices o mentales, no puedan manipular o interpretar los resultados del coagulómetro.

- La automonitorización no está indicada en pacientes (o cuidadores) que no estén motivados para ello.

\section{Conflicto de intereses}

Los autores declaran no tener ningún conflicto de intereses.

\section{Bibliografía}

1. January C, Wam S, Alpert J, et al., 2014 AHA/ACCI HRS. Guideline for the management of patients with atrial fibrillation: executive summary. A report of the American College of Cardiology/American Heart Association Task Force on Practice Guidelines and the Heart Rhythm Society. J Am Coll Cardiol. 2014;64:2246-80.

2. Wigle P, Hfin B. Update guidelines on outpatient anticoagulation. Am Fam Physician. 2013;87:556-66.

3. Nutescu E, Chuatrisora I, Hellenbart E. Drug and dietary interactions of warfarin and novel oral anticoagulants: an update. J Thromb Thrombolysis. 2011;31:326-43.

4. Higashi MK, Veenstra DL, Kondo LM, et al. Association between CYP2C9 genetic variants and anticoagulation-related outcomes during warfarin therapy. JAMA. 2002;287:1690-8.

5. Samsa GP, Matchar DB. Relationship between test frequency and outcomes of anticoagulation a literature review and comentary with implications for the design of randomized trials of patients self-management. J Thromb Thrombolysis. 2000;9:283-92.

6. Cosmi B, Palaren G, Mora, et al. Assesment of patient capability to self-adjust oral anticoagulant dose study on home use portable prothrombin time monitor. Haematologica. 2000;85:826-31.

7. Ansell J, Jacobson A, Levy J, et al. Guidelines for implementation of patients self-testing and patient self-management of oral anticoagulation. International consensus guidelines prepared by International Self-monitoring Association for Oral Anticoagulation. Int J Cardiol. 2005;99:37-45.

8. Garabedian-Ruffalo SM, Gray AR, Sax MJ, et al. Retrospective evaluation of a pharmacist-managed warfarin anticoagulation clinic. Am J Hosp Pharm. 1985;42:304-8.

9. Cortelazzo S, Emazzi G, Vieron P, et al. Thrombotic and hemorraghic complications in patients with mechanical heart 
value prosthesis attending an anticoagulation clinic. Thromb Haesmost. 1993;69:316-20.

10. Wilt VM, Gums JG, Ahmed Ol, et al. Pharmacy operated anticoagulation service: improved outcomes in patients on warfarin. Pharmacotherapy. 1995;15:732-79.

11. Chiquette L, Amato ME, Bussey HL. Comparison of anticoagulation clinic and usual medical care anticoagulation control, patient outcomes and health care costs. Arch Intern Med. 1998;158:1641-7.

12. Vander Besselaar AM. A comparison of INRs determined with a whole blood prothrombin time device and two international reference preparations for thromboplastin. Thromb Haesmost. 2000;84:410-2.

13. Horstkotte D, Piper C, Wiemer M, et al. Improvement of prognosis by home prothrombin estimation in patients with life-long anticoagulant therapy. Eur Heart J. 1996;17Suppl:230.

14. Sawick PT, For the working Group for the Study of Patient Selfmanagement of oral Anticoagulation. A structured teaching and self-management program for patients receiving oral anticoagulation. A randomized controlled trial. JAMA. 1999;281:145-50.

15. White RH, McCurdy SA, von Marensdorff H, et al. Home prothrombin time monitoring after the initiation of warfarin therapy. Ann Med. 1989;111:730-7.

16. Beyth RJ, Quinn L, Landefeld CS. A multicomponent intervention to prevent major bleeding complications in older patients receiving warfarin a randomized controlled trial. Ann Intern Med. 2000;133:687-95.

17. Nagler M, Bachman LM, Schmid P, et al. Patient selfmanagement of oral anticoagulation with vitamin $\mathrm{K}$ antagonists in every day practice: efficacy and safety in a nationwide longterm prospective cohort study. PLoS One. 2014;9:e 95761.

18. Kaatz S, Elston-Lafata J, Gooldy S. Anticoagulation therapy home and office monitoring evaluation study. J Thromb Thrombolysis. 2001;12:111.

19. Watzke HH, Forberg E, Svolba G, et al. A prospective controlled trial comparing weekly self- testing and self - dosing with the standard management of patients on stable oral anticoagulation. Thromb Haemest. 2000;83:661-5.

20. Okuyama Y, Matsuo M, Matsuo H, et al. Introduction of point-ofcare testing in Japanese outpatient clinics is associated with improvement in time in therapeutic range in anticoagulanttreated patients. Circ J. 2014;78:1342-8.

21. Matchar DB, Jacobson A, Dolor R, et al. Effect of home testing of international normalized ratio on clinical events. N Eng J Med. 2010;363:1608-20.

22. Garcia-Alamino JM, Ward AM, Alonso-Coello P, et al. Selfmonitoring and self-management of oral anticoagulation. Cochrane Database Syst Rev. 2010:CD003839.
23. Menenghelo ZM, Barroso CM, Lipopace IL, Cora AP. Comparison of the International normalized ratio levels obtained by portable coagulometer and laboratory in a clinic specializing in oral anticoagulation. Int J Lab Hematol. 2015;37:536-43.

24. Brouwer JL, Stovelaar H, Sucker C. The clinical impact of different coagulometers on patient outcomes. Adv Ther. 2014;31:639-56.

25. Thompson AN, Ragucci KR, Fermo D, Whitley HP. Evaluation of patients perceptions and outcomes related to anticoagulation point-of-care testing in ambulatory care clinics. Pharm Pract. 2009;4:213-7.

26. Caro JJ. An economic model of stroke in atrial fibrillation: the cost of suboptimal oral anticoagulation. Am J Manag Care. 2004; 10Suppl14:451-8.

27. Muller E, Bergemann R, The GELIA study group. Economic analysis of bleeding in thromboembolic sequelae after heart valve replacement (GELIA 7). Eur Heart J. 2001; 3 Suppl Q:Q 65-69.

28. Sharma P, Scotland G, Cruickshank M, et al. The clinical effectiveness and cost-effectivenness of point-of-care tests (Coagucheck system, INRatio 2 PT/INR monitor and Protime Microcoagulation system) for the self-monitoring of the coagulation status of people receiving long-term vitamin $\mathrm{K}$ antagonist therapy, compared with standard UK practice: systematic review and economical evaluation. Health Technol Assess. 2015;19:1-172.

29. Alfonso-Coello P, Zhou Q, Guyatt G. Home-monitoring of oral anticoagulation vs. dabigatran. An indirect comparison. Thromb Haemost. 2012;108:647-53.

30. Fitzmaurice DA, Murray ET, Gee KM, et al. A randomised controlled trial of patient self management of oral anticoagulation treatment compared with primary care management. J Clin Pathol. 2002;55:845-9.

31. Blomfield HE, Krause A, Greer N, et al. Meta-analysis: effect of patient self-testing and self-management of long-term anticoagulation on major clinical outcomes. Ann Int Med. 2011;154:472-82.

32. Jenner KM, Simmons BJ, Delate $T$, et al. An education program for patient self-management of warfarin. Perm J. 2015;19:33-8.

33. Ansell J, Becker D, Andreww, et al. Accurate and precise prothrombin time measurement in a multicenter anticoagulation trial employing patient self-testing. Blood. 1995; 86Suppl: 864a.

34. Jennings I, Kitchen D, Keeling D, et al., On behalf of the BCSH Committee. Patient self-testing and self-management of oral anticoagulation with vitamin $\mathrm{K}$ antagonists: guidance from the British Committee for Standars in Haematology. Br J Haemt. 2014;167:600-7. 